\title{
Outcomes, what outcomes?
}

\author{
Derek Richards \\ Editor, Evidence-based Dentistry \\ Evidence-Based Dentistry (2005) 6, 1. doi:10.1038/sj.ebd.6400313
}

One of the first stages of the evidencebased approach is to formulate a clinical question. The standard way to do this is by using the well-established PICO (problem, intervention, comparison, outcome) format, as described by Richardson et al. ${ }^{1}$ This approach is also taken to define the problem in systematic reviews because poorly focused questions lead to unclear decisions about what research to include and how to summarise it.

Regular readers will be aware that many of the systematic review summaries that we publish in Evidence-based Dentistry find that the available evidence is not strong enough to answer the questions posed by the reviewers. One of the strengths of a well-conducted systematic review, the identification of all the available literature on a topic, can be one of its frustrations because the quality of the studies is not strong enough to answer the question posed without bias. Consequently, it tells us not only how little we do know but how much we do not know.

As well as the frequent problems with the quality of study-design or of reporting, which both cause difficulty when conducting a systematic review, there is a further difficulty. This is related to the outcome or outcome measures used (a good classification of the types of outcomes available is given by Bader and Ismail). ${ }^{2}$ For example, there are a large number of indices for expressing tooth decay and periodontal disease, or potential outcomes in temporomandibular disorders. This causes problems when conducting meta-analyses. Sutherland and Matthews ${ }^{3}$ have discussed the issues caused by this, highlighting some of the lessons learned while preparing systematic reviews for clinical guidelines. Hujoel ${ }^{4}$ has also argued that the "lack of a rigorous scientific basis for the measurement of periodontitis has led to changing opinions as to what measures should be used to assess periodontal treatment efficacy and how to interpret changes". Hujoel highlights too the difference between true end points, which are tangible to the patients and directly measure how a patient feels, functions or survives (eg, tooth loss or pain or oral health-related quality-of-life measurements), and surrogate end points: the latter are intangible to the patient, such as changes in probing attachment level or gingival crevicular fluid level.

In dentistry our focus tends to be on surrogate end points or outcomes although there is an increasing focus on oral health-related quality of life measures. Hujoel does support the use of surrogate end points when two conditions are met, however:

- Informativeness: is there evidence that the surrogate predicts the true end point? - Specificity: is the treatment effect on the true endpoint mediated through the surrogate end point?

Although the focus of Hujoel's paper is periodontal disease, many of his arguments are just as relevant to other fields of dental research.
In the field of dental caries, work has started on the development of "ICDAS": an international system for caries detection and assessment. ${ }^{5}$ The aim of ICDAS is to facilitate caries epidemiology, research and appropriate clinical management. This has the potential to help greatly when combining studies on dental caries into a meta-analysis in the future. There is the scope for similar developments in other fields within dentistry. There is the opportunity for organisations such as the International Association for Dental Research and the World Dental Federation, along with specialist associations, to lead the way to a greater consistency in the outcomes we measure in dentistry. Without this uniformity we are not making the most of the original research that is being carried out and we may not realise the full potential of results combined into systematic reviews.

1. Richardson WS, Wilson MC, Nishikawa J, Hayward RS. The well-built clinical question: a key to evidence-based decisions. ACP J Club 1995; 123:A12-A13.

2. Bader JD, Ismail Al. A primer on outcomes in dentistry. J Publ Health Dent 1999; 59:131-135.

3. Sutherland SE, Matthews DC. Conducting systematic reviews and creating clinical practice guidelines in dentistry: lessons learned. J Am Dent Assoc 2004; 135:747-753.

4. Hujoel PP. Endpoints in periodontal trials: the need for an evidence-based research approach. Periodontology 2000 2004; 36:196-204.

5. Pitts N. 'ICDAS' - an international system for caries detection and assessment being developed to facilitate caries epidemiology, research and appropriate clinical management. Commun Dent Health 2004; 21:193-198. 